\title{
Mechanism for Shish Formation under Shear Flow: An Interpretation from an in Situ Morphological Study
}

\author{
Bo Shen, ${ }^{\dagger}$ Yongri Liang, ${ }^{\dagger}$ Julia A. Kornfield, ${ }^{\dagger}$ and Charles C. Han* ${ }^{\dagger}$ \\ †State Key Laboratory of Polymer Physics and Chemistry, Joint Laboratory of Polymer Science and Materials, \\ Beijing National Laboratory for Molecular Sciences, Institute of Chemistry, Chinese Academy of Sciences, Beijing 100190, P. R. China \\ ${ }^{\ddagger}$ Division of Chemistry and Chemical Engineering, California Institute of Technology, Pasadena, California 91125, United States
}

Supporting Information

ABSTRACT: Isotactic polypropylene has been systematically studied with in situ optical microscopy to obtain a real space view of the morphology evolution of shear induced crystallization as a function of shear rates and shear time to elucidate the mechanism of shish formation. The critical shear time for inception of shish formation on the shear rate have provided an important understanding of the molecular and entangled network relaxation

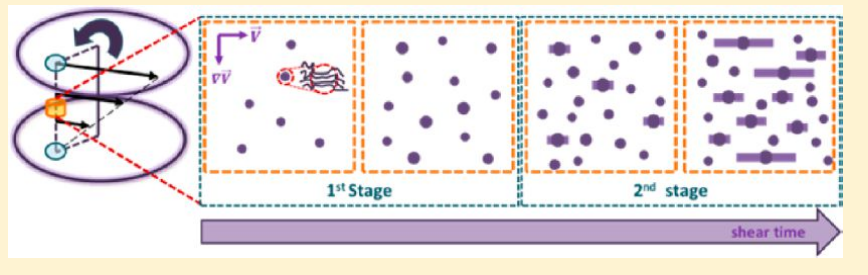
in relation to the shish formation. Also the observation of a typical shear time dependent comet like shish-kebab structure formation on the interface and the morphological growth of the shish from a fiber inside our sample have led to a new hypothesis that the shish is formed through a multiple discrete steps instead of forming directly to the final most stable state, this means that a transition state may be existed before shish growth. Two steps shear experiments with various time intervals between each step were designed to verify our proposed transition state mechanism, which can be observed much directly and obviously through real-space morphology, especially at low shear rate with long shear time. Another time dependent relaxation time is introduced base on shear rate dependent experiment after the first step shear and has close relationship with the existence of the critical shear time. A general framework for the shish formation has been established which can capture all the observed morphological features well, including the existence of the critical shear time at a given shear rate.

\section{INTRODUCTION}

About two-thirds of commercial synthetic polymers are semicrystalline polymers. During processing, the polymer melts are subjected to complicated flow field that often accelerate crystallization kinetics by orders of magnitude and dramatically influence the semicrystalline morphology. Anisotropic "shishkebab" structure is commonly induced under such processing conditions, in contrast to the spherullitic structure formed under quiescent conditions. So shear induced crystallization has drawn considerable attention due to both scientific significance and industrial value, including experimental, ${ }^{1-8}$ theoretical, ${ }^{9-12}$ and computational studies. ${ }^{13-15}$ Some features of the transition to highly oriented structure are widely accepted, such as the increase in shish formation with increasing of shear rate. ${ }^{16,17}$ However until now the quantitative effects of important control parameters (shear rate, shear stress, time, molar mass, and its distribution) are not fully understood. Here we give particular attention to the effect, shear time, which has not yet received enough attention in the literature. Because of the coil-stretch transition concept introduced by Keller which originated from the description of the abrupt distortion of polymer chain shape in dilute solution under the shear field proposed by De Gennes, ${ }^{18}$ the high molecular weight chains are consider to have an important role in promoting the shish nucleation, thus most of studies in recent years have focused on revealing the way in which these chains affect the shish-kebab formation.,19-22 Although adding the long chain polymers are indeed able to effectively promote the formation of shish-kebab, their concentration must be above the overlap concentration ${ }^{23}$ and effect will saturate when long chain pervade the entire volume ${ }^{24}$ as suggested by Kornfield which means its effect is cooperative rather than a single chain effect and their further experiment show the fraction of long chain molecular in the shish matches that in the bulk. ${ }^{25}$ These results are not compatible with the original idea of coilstretch transition model in which the shish are mostly composed of high molecular weight component. The impact of Keller's model which has been discussed by many authors is profound, but the transplanting of De Gennes' theory from solution to bulk is questionable. There are two important considerations as stated by Janeschitz-Kriegl, ${ }^{26}$ one is that in the relaxation experiment, the oriented long molecules which disperse among the short ones will take more time to diffuse in the direction perpendicular to the stretched chain, so these chains will relax before getting together. The other is that the length of the shish can reach the macroscopic orders of magnitude from observation and is several hundred times the length of the single chain. Because of these arguments and experimental facts, people have to modify Keller's idea and try to establish more reasonable models. An aggregation type mechanism first proposed by Hoffman in polymer solution suggested that shish may be formed by discrete steps, ${ }^{27}$ the

Received: November 20, 2012

Revised: January 23, 2013

Published: February 11, 2013 

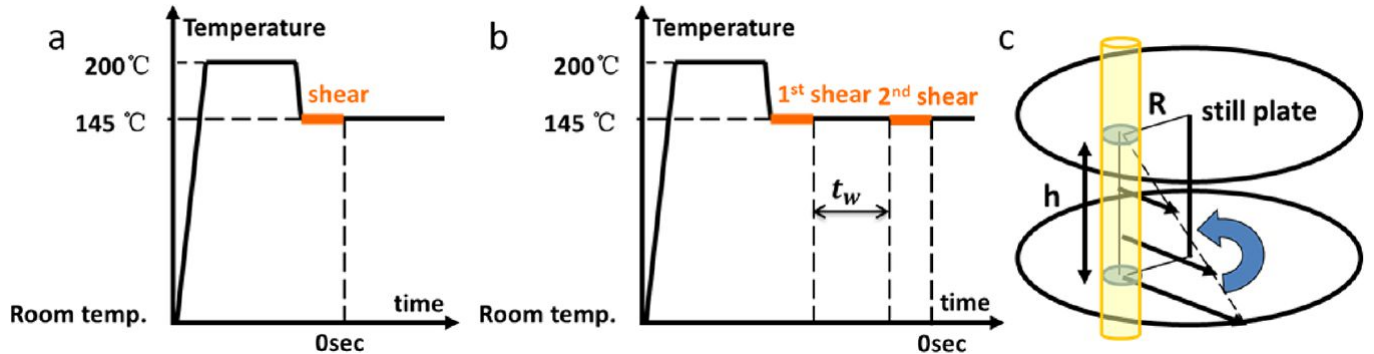

Figure 1. Temperature and shear protocol for (a) "one step" shear and (b) "two step" shear-induced crystallization experiments performed in (c) a parallel plate shear device mounted in a microscope with the stationary plate on top and the rotating plate below. In most experiments, the focal plane is at the midplane between the parallel plates; where noted, the focus is moved to the polymer-plate interface at the top or bottom plate.
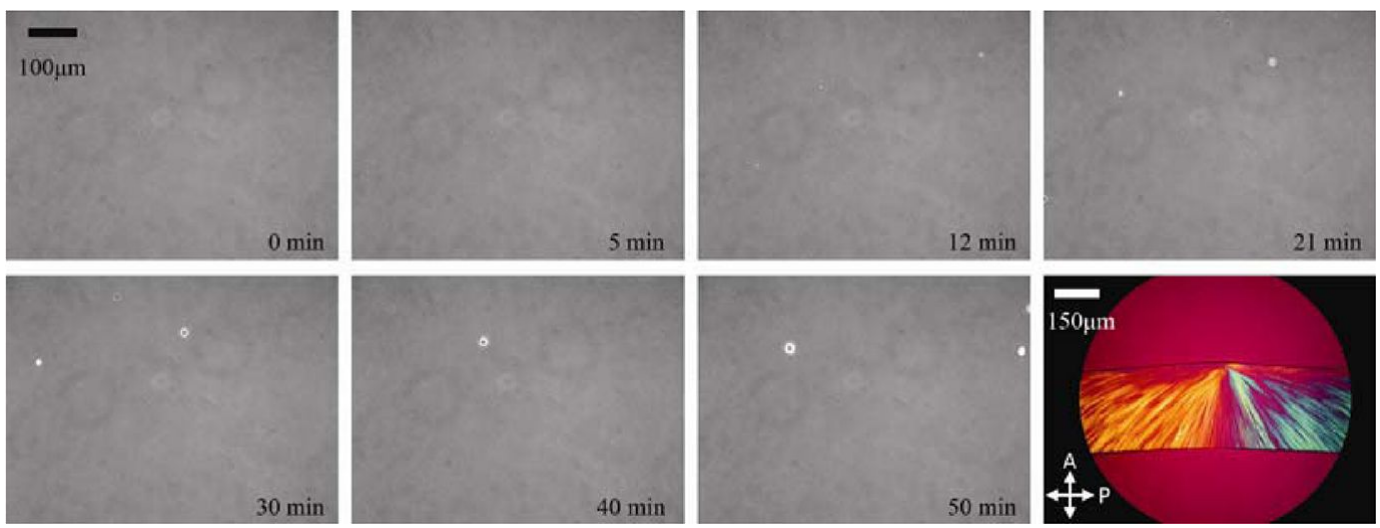

Figure 2. In situ time evolution phase contrast optical microscope (PCOM) image of iPP sample crystallized in quiescent condition at $145^{\circ} \mathrm{C}$ and the ex situ polarized optical micrograph of the cross section of fully crystallized sample with full-wave retarder (the slow axis aligns from the top right to the bottom left). The order of interference color changes due to the geometry of the chain axis and slow axis of wave plate.

multiple micellar crystals formed on a flow-elongated molecule during the shear would aggregate along the flow direction into oriented threads as the chain segments between the nuclei were pulled taut. ${ }^{28-30}$ Alternatively, the stretched network model proposed that the deformed network instead of a single chain is responsible for the shish formation. ${ }^{31-34}$ While Janeschitz-Kriegl believed that for the formation of such a long shish there exists only one possibility based on the two facts mentioned above: through the growth mechanism. From some local alignments of "athermal nuclei" with the fringe micelle shape which might be already existed in the quiescent melt below the melting temperature, the stretched long molecules within certain critical contact region will then touch these aggregates when they pass by along the flow direction and start to grow into threads. According to this interpretation, further argument was made that the process must depended on the even function of the shear rate as required for independence of shear direction and also for the simplicity only the square term was used which could be considered as the second invariant of velocity gradient tensor in the Couette flow. ${ }^{4}$ The more physical description of the square shear rate dependence of this process is chosen to be the specific work which was verified by Mykhaylyk as the critical control parameter for shish formation. ${ }^{30,35}$ Evidence in favor of the idea for growth mechanism according to Janeschitz-Kriegl is that the total length of all the shish per unit volume is extraordinary high which can be qualitatively described by the fourth power dependence of the shear rate.

The correct interpretation of experimentally observed effects of control parameters (e.g., shear time, molecular weight, etc.), depends on the correct understanding of the mechanism of shish structure formation; therefore, a model that can correctly describe shish formation is important and needed. Experimental results in real space and real time can accelerate theoretical progress by discriminating among proposed models and mechanism. In contrast to X-ray scattering and rheological measurements, for example, that probes ensemble properties of a system (averaging over many crystalline objects formed at different times after inception of shear). There is no thermodynamic argument that all nuclei are formed exactly at the end of the shear time imposed on the system, while in fact the nuclei could be formed during the shear. ${ }^{36}$ Here we use real time optical microscopy to provide real space morphological information, including images of the formation and growth process of individual nuclei and shish during shear flow. The morphological development of individual shish as a function of shearing conditions will give reasonable answers about how the shish is formed under shear flow and the interpretation of why the critical shear time existed for the formation of shish with a fixed shear rate.

\section{EXPERIMENTAL SECTION}

Material. Isotactic polypropylene (iPP) $\left(M_{\mathrm{w}}=3.4 \times 10^{5} \mathrm{~g} / \mathrm{mol}\right.$, $\left.M_{\mathrm{w}} / M_{\mathrm{n}}=3.7\right)$ was provided by Yanshan Petrochemical Corp., Inc.

Instrument. A commercial parallel plate shearing hot stage Linkam CSS-450, which provides control of shearing conditions, was used with optical microscope (Nikon E600POL) to study shear induced crystallization. The sample was held between two quartz plates and was sheared by rotation of the bottom plate with top plate stationary (Figure $1 b$ ).

Parallelism of the plates was readily checked because the sample was easy to remove at the end of each test. The thickness was measured at various positions on the resulting iPP disk and was found to vary by less than about $1 \%$. 

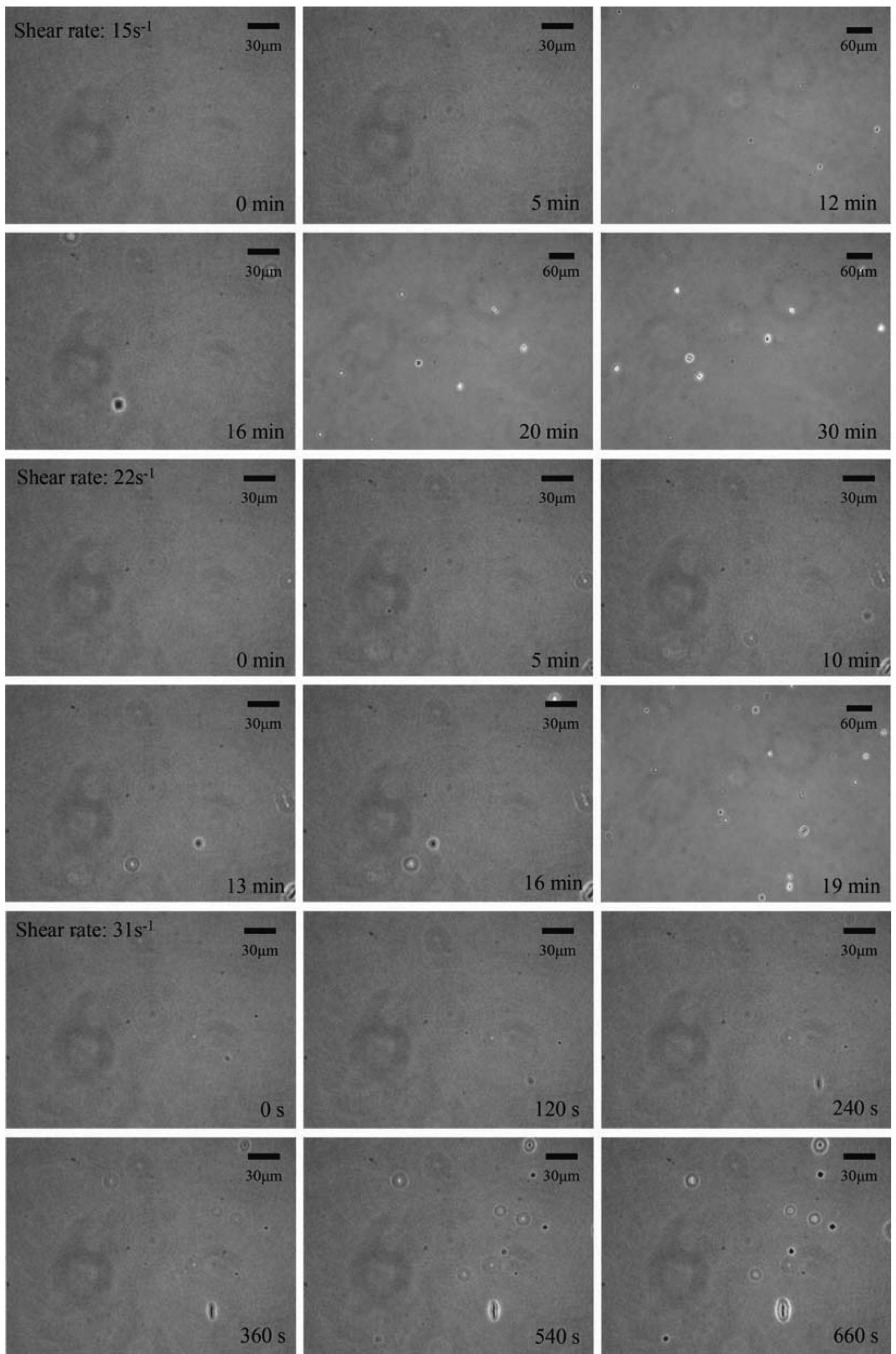

Figure 3. In situ time evolution of PCOM morphology of iPP sample under various shear rates $\left(15 \mathrm{~s}^{-1}, 22 \mathrm{~s}^{-1}\right.$, and $\left.31 \mathrm{~s}^{-1}\right)$ for constant shear time $1 \mathrm{~s}$ at the temperature of $145^{\circ} \mathrm{C}$. 

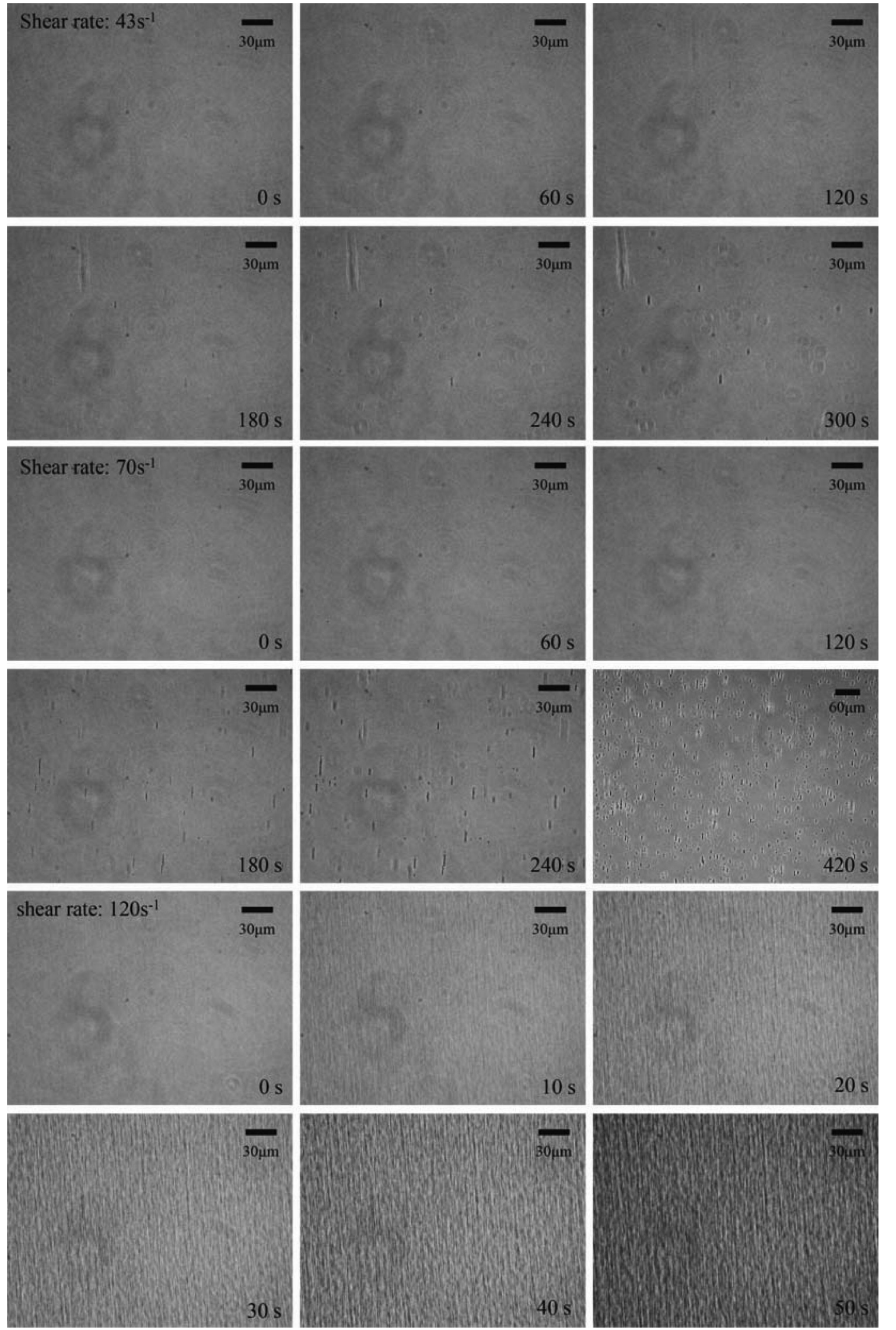

Figure 4. In situ time evolution of PCOM morphology of iPP sample under various shear rates $\left(43,70\right.$, and $\left.120 \mathrm{~s}^{-1}\right)$ for constant shear time $1 \mathrm{~s}$ at the temperature of $145^{\circ} \mathrm{C}$. 

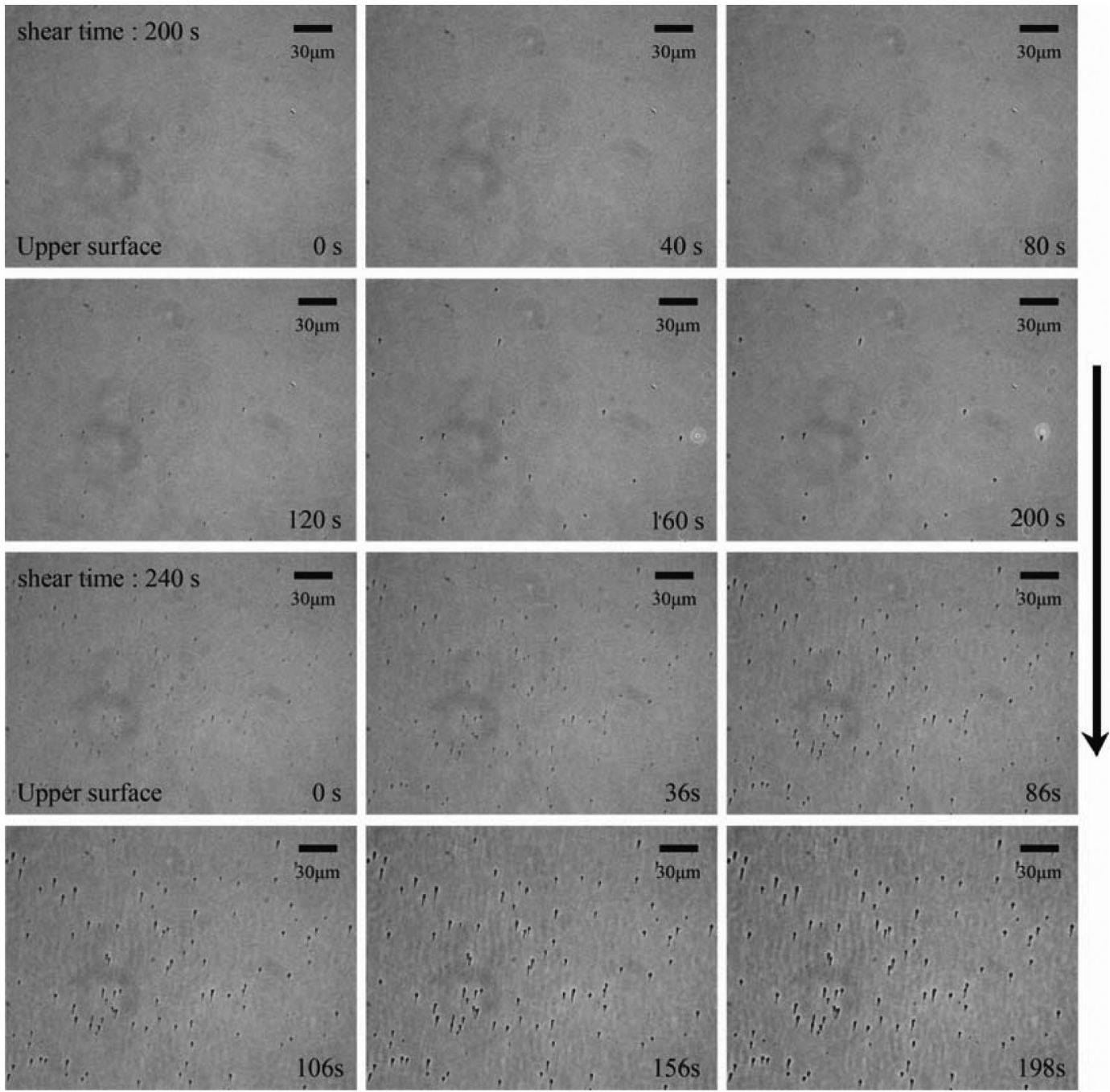

Figure 5. In situ time evolution of phase contrast optical micrographs of comet like shish-kebab morphology formed on the upper surface due to weak slip happened on the wall under the apparent shear rate $0.5 \mathrm{~s}^{-1}$ with different shear times of 200 and $240 \mathrm{~s}$ at a temperature of $145^{\circ} \mathrm{C}$. The black arrow indicates the flow direction.

Experimental Details. The iPP sample was initially held at $200{ }^{\circ} \mathrm{C}$ (approximately $35^{\circ} \mathrm{C}$ above the melting temperature measured by DSC, MettlerToledo-822e) for 8 min to erase thermal history effects, then quenched to $145^{\circ} \mathrm{C}$ with the cooling rate of $30^{\circ} \mathrm{C} / \mathrm{min}$. This protocol was validated using the following test: a sample was sheared to induced shish-kebabs at $145{ }^{\circ} \mathrm{C}$, then heated to $200{ }^{\circ} \mathrm{C}$,held for $8 \mathrm{~min}$, then cooled to $145^{\circ} \mathrm{C}$ again and only normal quiescent crystallization took place. In our experiment, when the sample is cooled and just after reaching the crystallization temperature, the sample was subjected to the shear with specified shear rate for certain shear time (Figure 1a).

From one experiment to the next, the sample thickness varied by as much as $10 \%(100 \pm 10 \mu \mathrm{m})$. Unless it is mentioned in the text (for Figure 5 as well as for Figures 9 and 10), the microscope is focused in the midplane of the sample. Experiments were performed at this temperature $\left(145^{\circ} \mathrm{C}\right)$ because all nucleation observed were started from the melt-substrate interface (Figure 2), so the homogeneous nucleation maybe negligible. Both of the quartz plates used in each experiment were carefully cleaned to suppress the wall slip following the procedure of our previous study. ${ }^{37}$

\section{RESULTS AND DISCUSSION}

One Step Shear Experiments. To better understand the effect of shear flow on the morphology of polymer crystallization, we first use the short-term shearing protocol of Janeschitz-Kriegl to study both the effects of shear rate (Figures 3 and 4) and shear time (Figures 5-8) and compare them with quiescent crystallization of iPP at the same temperature (Figure 2).

Effects of Shear Rate. Similar to the classification scheme of van Meerveld et al., ${ }^{38}$ however, we find there are four regimes during the morphology evolution as a function of shear rate, obviously the boundary will depend on the shear time (here using the constant shear time of 1s). At low shear rate (e.g., $0.5 \mathrm{~s}^{-1}$ ), based on the result shown in Figure 5, it is as expected that the shearing has almost no effect on crystallization kinetics. As the shear rate increased, the crystallization kinetics changed slightly from the quiescent crystallization case, a little more nuclei were induced inside the sample $\left(15 \mathrm{~s}^{-1}\right.$ Figure 3$)$. At intermediate shear rates (e.g., between 22 and $43 \mathrm{~s}^{-1}$ ), the number of nuclei increased and the induction time decreased (e.g., to less than $1 \mathrm{~min}$ at the shear rate of $43 \mathrm{~s}^{-1}$ ) (Figure 3 and 4). Then, above a critical shear rate of approximately $56 \mathrm{~s}^{-1}$, shear induced formation of some cylindrulites (characteristic of shish kebab structure) dispersed among small "point-like" nuclei (see Figure 4 and also Figure 12). There is no sharp boundary between spherulitic and cylindrulitic structure formation, the further increase of the shear rate just gradually increases the ratio of cylindrulites to spherulites, together with increased nucleation density and decreased induction time. When the shear rate 

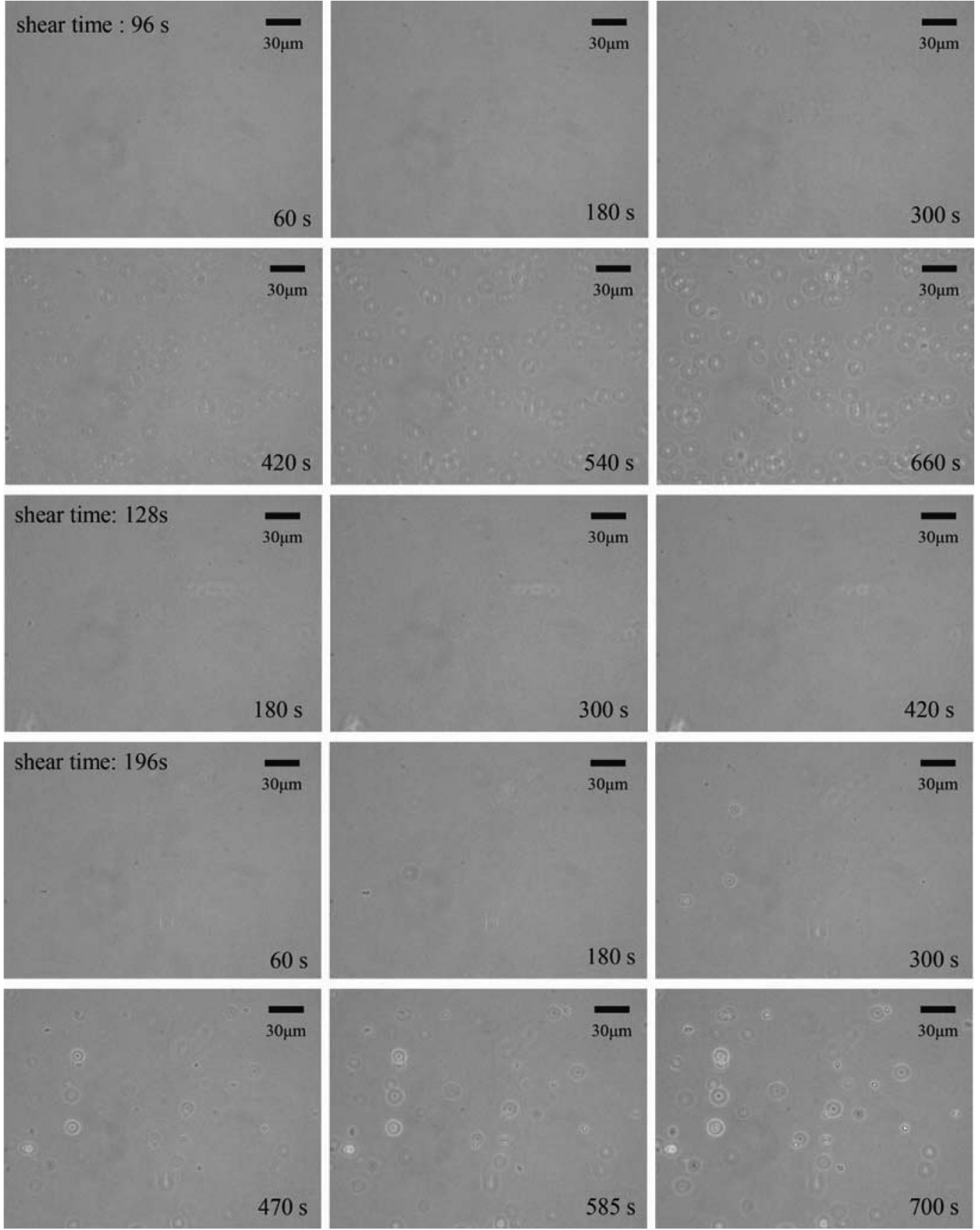

Figure 6. In situ time evolution of phase contrast optical micrographs of iPP sheared at the temperature of $145^{\circ} \mathrm{C}$ under the shear rate $3.6 \mathrm{~s}^{-1}$ with a shear time of 96,128 , and $196 \mathrm{~s}$. The microscope is focused at the middle of the sample.

reached approximately $120 \mathrm{~s}^{-1}$ which is above the threshold shear rate for inducing a highly oriented structure: after $1 \mathrm{~s}$ of shear, the cylindrulites appeared within 10s and quickly filled the entire field of view (Figure 4). The cylindrulites seem to grow to impingement quickly (note that images at 20,30, 40 and 50s are almost indistinguishable)

Effect of Shear Time. The dependence of the morphology on shear time is different in the various regimes (For example: the classification of shear rate earlier is under the shear time of $1 \mathrm{~s}$ ). As expected, when the shear rate is too low to enhance nucleation, the effect of shearing time is weak. For example, if a low shear rate is applied $\left(0.5 \mathrm{~s}^{-1}\right.$ in Figure 5), even if the shear time was increased to 200 and 240 s, shearing had no effect on the nuclei formation inside the sample, although nuclei formation on the interface was enhanced. ${ }^{37}$ Similar result were observed for a shear rate of $3.6 \mathrm{~s}^{-1}$ and a shear time of $96 \mathrm{~s}$ (the microscope was focused in the middle of the sample, so nuclei on interface are seen out of focus), while for shear time as long as $196 \mathrm{~s}$, the number of nuclei induced inside of sample was still very little, however, slowly increasing. In the higher shear rate regime (regime three and regime four) in which a combination of cylindrulites and spherulites are observed, the shearing time has a pronounced effect. For example, for a shear rate $80 \mathrm{~s}^{-1}$, after cessation of the short-term shearing of $1 \mathrm{~s}$ (Figure 8), cylindrulites of short length appear within $60 \mathrm{~s}$, dispersed among small nuclei. Increasing the shear time to $2 \mathrm{~s}$ significantly increases the density of cylindrulites due to both a greater number per unit volume and a greater length. 

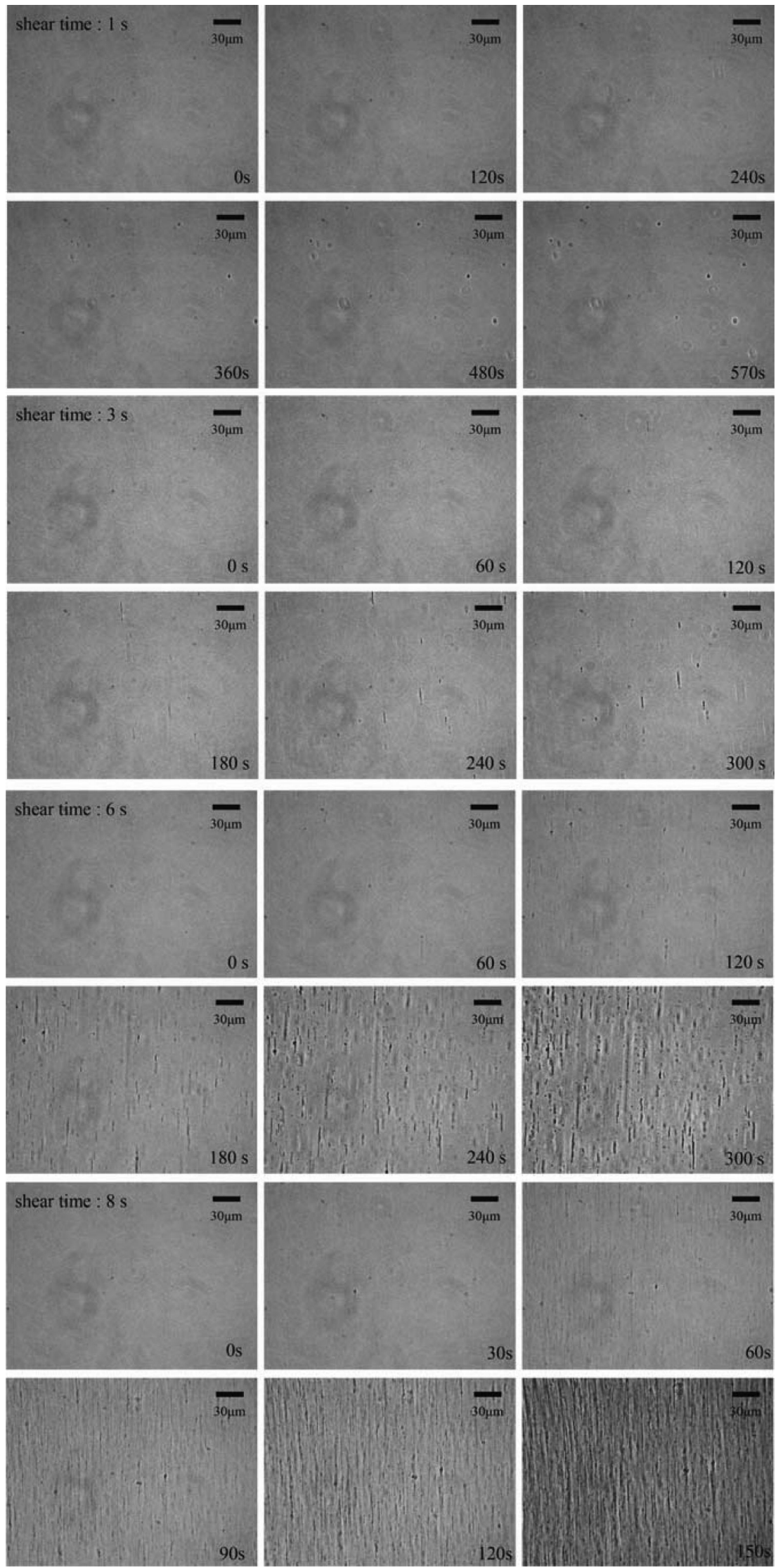

Figure 7. In situ time evolution of phase contrast optical micrographs of iPP sheared at the temperature of $145^{\circ} \mathrm{C}$ under the shear rate $36 \mathrm{~s}^{-1}$ with a shear time of $1,3,6$, and $8 \mathrm{~s}$. 

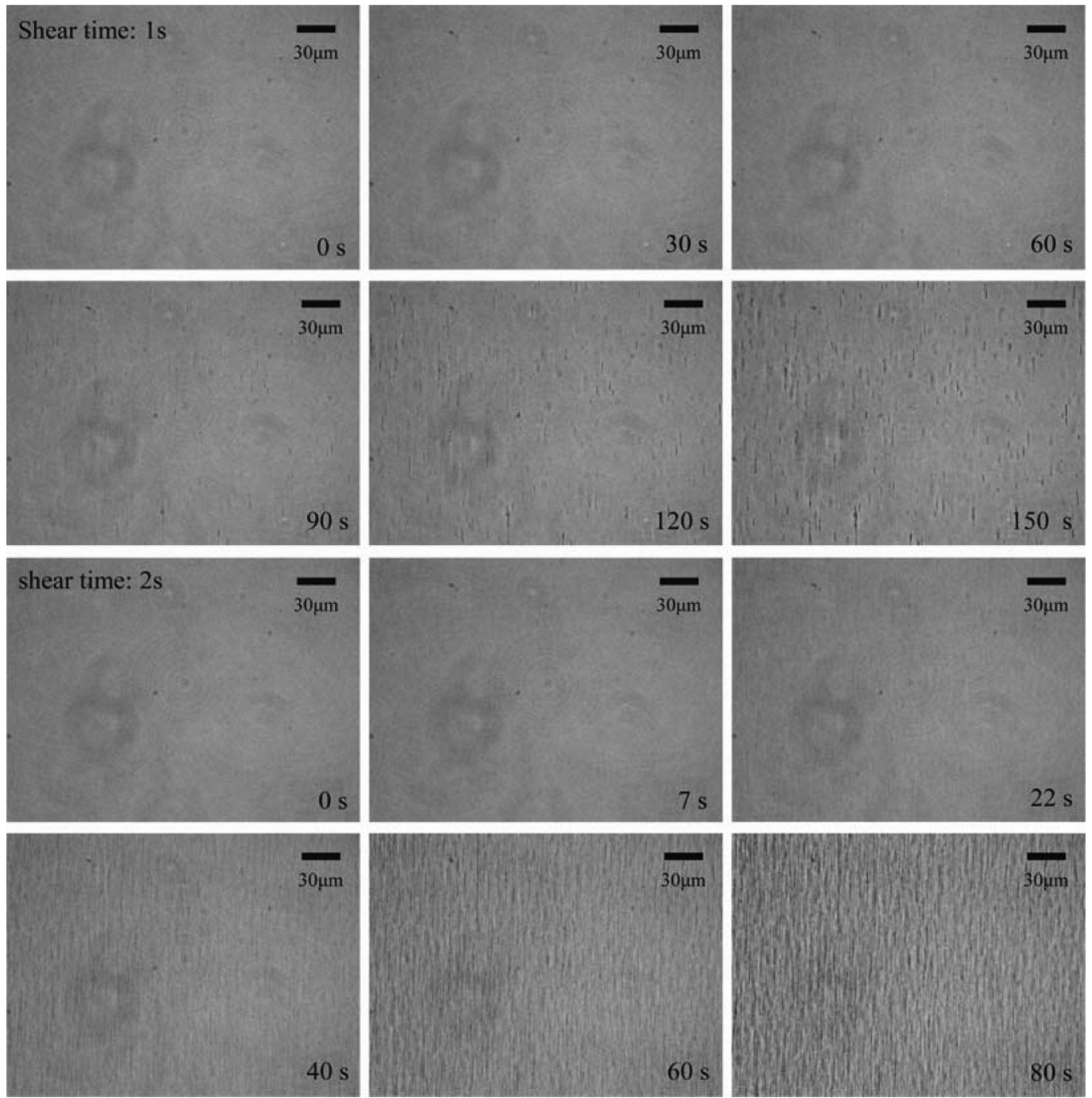

Figure 8. In situ time evolution of phase contrast optical micrographs of iPP sheared at the temperature of $145^{\circ} \mathrm{C}$ under the shear rate $80 \mathrm{~s}^{-1}$ with a shear time of 1 and $2 \mathrm{~s}$.

At intermediate shear rates, which are the focus of this study, cylindrulites only formed above a critical shear time that depends on the shear rate. Although people have known this fact for many years, ${ }^{2,17}$ however, the underlying physics still remain elusive. The specific example shown in Figure 7 is representative of the range of shear rate from 31 to $51 \mathrm{~s}^{-1}$. For "short" shear times, only point-like nuclei were induced and their density increased with increasing shear time (the rate of nucleation depended on the shear rate applied). For shear times above a critical value, some cylindrulites formed, giving rise to shear time dependent morphology evolution progressing from spherulitic to cylindrulites dispersed in spherulites, which is similar to the shear rate dependence at fixed shearing time, and finally to completely cylindrulitic morphology. If the shish-kebab could be induced, which means the shear rate we applied is above the critical value of disentanglement rate or the relaxation rate of entangled polymer of the shish forming polymers. Then the physics of why we still needs some shear time rather than instantaneously to form such a shish structure must be a key issue in understanding the critical shear time and the critical shear rate.

Obviously, this shear time is one of the control parameters which need to be seriously considered to achieve a comprehensive understanding of the shish-kebab formation mechanism.
We cannot delineate the mechanism from the morphological data so far, some further experiments have been designed and carried out to give a better physical picture which will be discussed as follows.

Two Step Shear Experiments. Motivation. The one step experiment highlight two important phenomena that are particularly important for understanding the shear induced shish-kebab structure. The first is the morphology of the shishkebab formed on the polymer-substrate interface, which is due to the wall slip as have been discussed in our previous study. ${ }^{37}$ Here we discovered that the typical shish-kebab morphology at different shear time on the upper and lower interface clearly shows that the shish grow out from nuclei that located at the polymer-plate interface (Figure 5). With time, the morphology becomes clearer as lamellae grow out from the precursors and make them visible: the shish extend only in one direction form nuclei on a given plate and the direction on one interface is opposite to the other, corresponding to the direction of the flow relative to a nucleus fixed on the plate (Figure 9). Figures 9 and 10 represent an extreme case; we can observe a similar result as shown in Figure S1 in the Supporting Information.

The second experimental result was found when foreign impurities were present inside the sample: shish-kebabs could be 


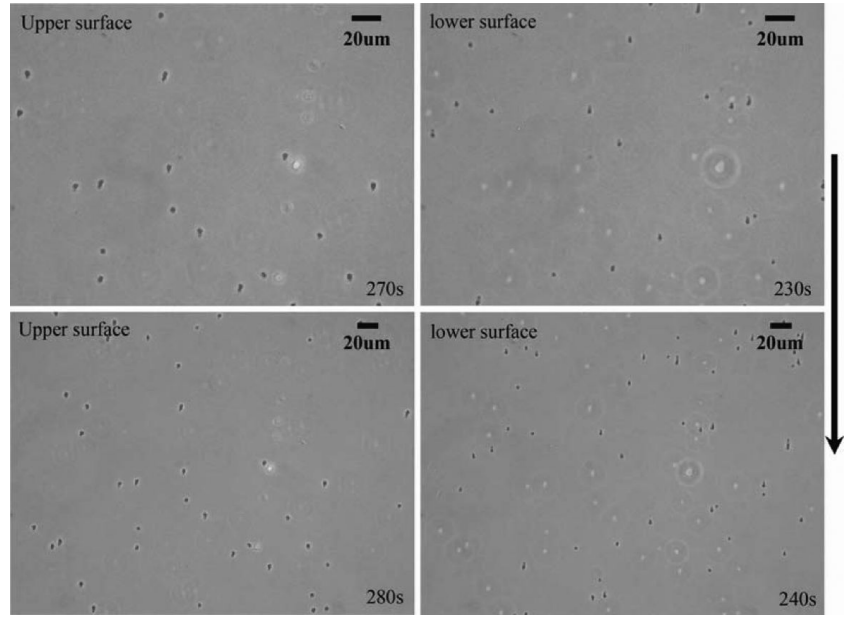

Figure 9. Phase contrast optical images of morphology formed at the polymer-substrate interface due to weak slip happened on the wall at the temperature of $145{ }^{\circ} \mathrm{C}$ under the shear rate $0.5 \mathrm{~s}^{-1}$ with the shear time $200 \mathrm{~s}$. The left column is the morphology at upper interface, and the right is that at the lower interface. The black arrow indicates the flow direction.

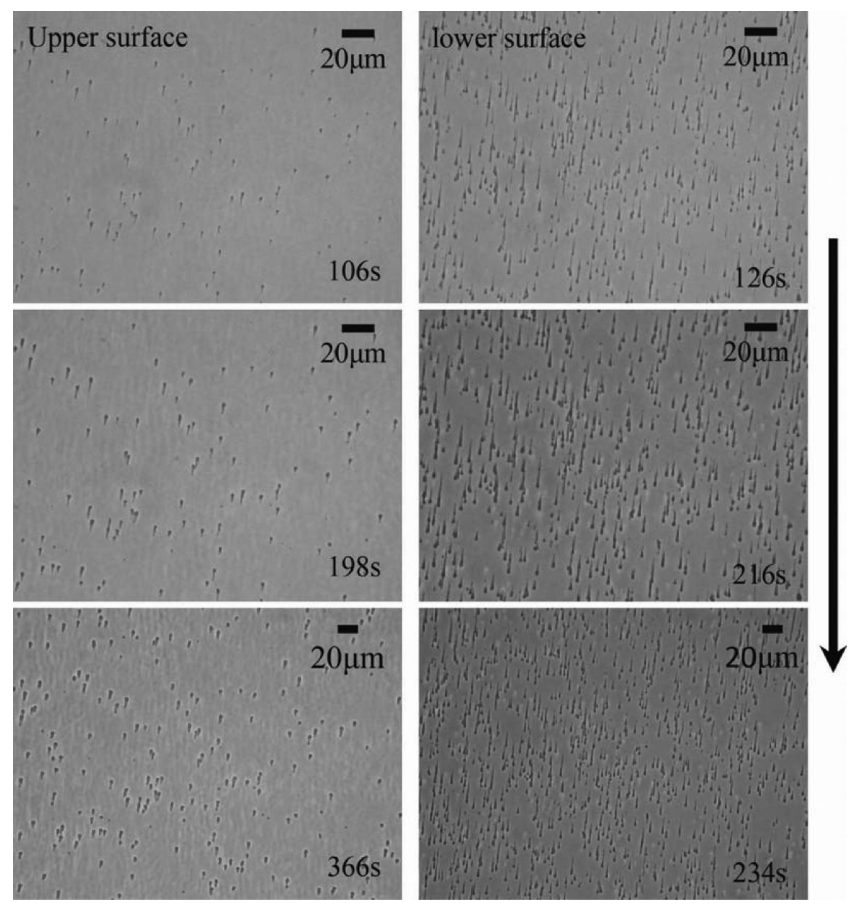

Figure 10. Phase contrast optical picture of the morphology formed at the polymer-substrate interface due to weak slip happened on the wall at the temperature of $145^{\circ} \mathrm{C}$ under the shear rate $0.5 \mathrm{~s}^{-1}$ with the shear time $240 \mathrm{~s}$. The left column is the morphology at upper interface, and the right is that at the lower interface. The black arrow indicates the flow direction.

easily induced from these external particles under a shear rate that is lower than the critical shear rate for the formation of shish-kebabs (e.g., shearing at $2 \mathrm{~s}^{-1}$ for 20 s, Figure 11). We also observed that no other nuclei were formed in region far away from these impurities (Figure 11a). When, by chance a fiber from lens paper was left inside the sample, we found that almost all the shish started from the fiber and apparently grew out from it on both sides (Figure 11c). In addition to the formation of apparently continuous threadlike precursors, "trains" of small
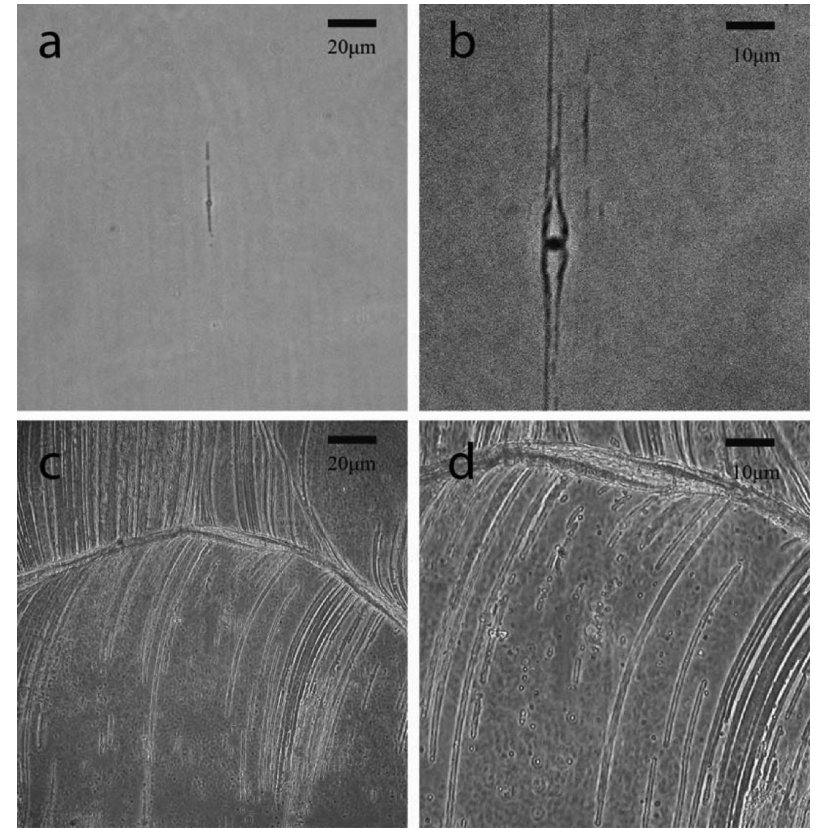

Figure 11. Representative phase contrast optical image of a typical shish-kebab morphology when there are foreign impurities inside of the sample: (a) the dust particle inside of the sample; (b) the much bigger dust particle with shish-kebab growth on both sides; (c) a fiber from lens paper, showing shearing at $2 \mathrm{~s}^{-1}$ for $20 \mathrm{~s}$; (d) enlarged image of a part of part c.

precursors were also seen along lines parallel to the threads (Figure 11d).

Together, the comet-like morphology of the shish-kebabs grew from nuclei on the interface and the morphology of shishkebabs that started from a foreign impurity influenced our thinking about the shish formation mechanism. Once a nucleus forms during shear (e.g., on the polymer substrate interface), then shish may start to grow from it by the same process as those that start on the foreign particles. To test the hypothesis that point-like nuclei function as the initial seed from which shish start to grow, we performed a carefully designed series of "two step shear" experiments.

Selection of Experimental Conditions. The results of single step shear experiments were used to select one special condition $\left(\dot{\gamma}, t_{s}\right)$ that gives mainly spherullites (i.e., flow induced precursors are short and far apart) ${ }^{39}$ and such that doubling the shearing time results in predominantly cylindrulitic morphology. Specifically, we selected $\left(\dot{\gamma}=56 \mathrm{~s}^{-1}, t_{s}=1 \mathrm{~s}\right)$, which induces short, widely spaced precursors (Figure 12); when the shear time is doubled ( $2 \mathrm{~s}$ of continuous shear, corresponding to zero time interval between two shear times of $1 \mathrm{~s}$ each, Figure 14, bottom), almost all precursors are threadlike. Then we split the doubled shear time experiment into two equal parts separated by a quiescent "waiting time" $t_{w}$ that was varied from 0 to $240 \mathrm{~s}\left(t_{w}\right.$ in Figure $1 \mathrm{~b}$ ). If our hypothesis is correct, then nuclei formed during the first step will alter flow-induced nucleation during the second step of the shear experiment.

First Step of Shearing. The time evolution of the morphology after the first step of shearing with the shear rate of $56 \mathrm{~s}^{-1}$ and shear time of $1 \mathrm{~s}$, indicates that the majority of flow induced precursors are less than $10 \mu \mathrm{m}$ long and spaced more than $20 \mu \mathrm{m}$ apart (Figure 12); this is deduced from the structures that become visible due to the overgrowth of kebabs, which gives readily visible structures after approximately 200s and corresponding faint 

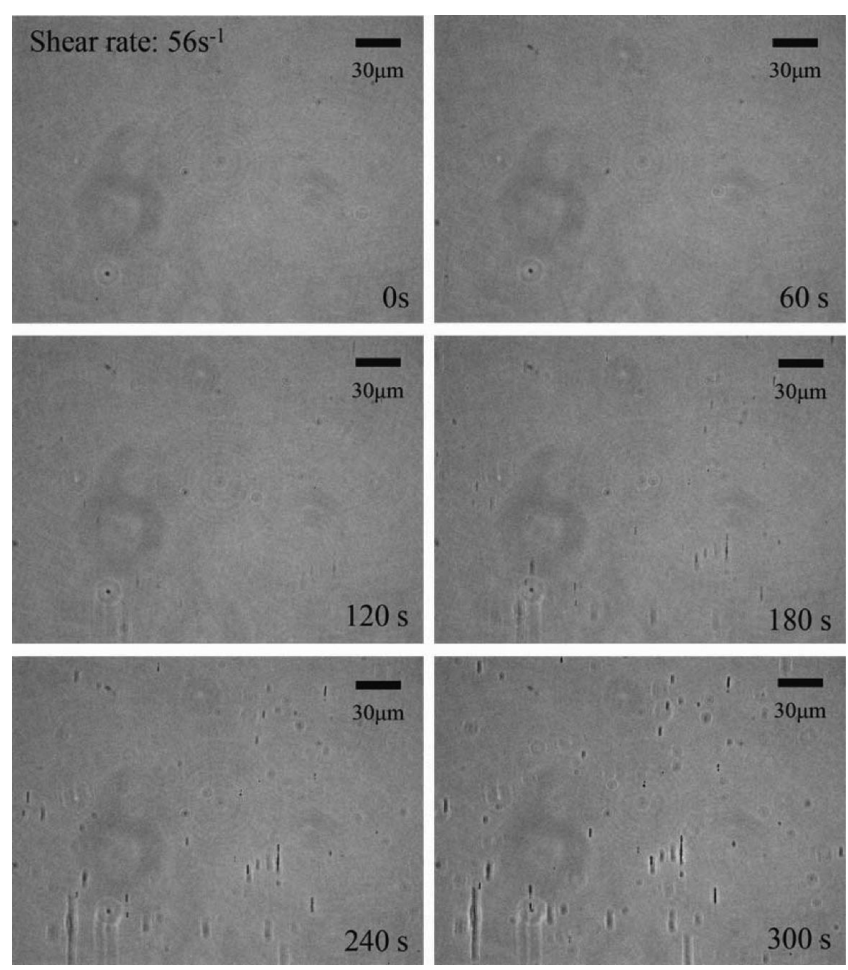

Figure 12. In situ time evolution of phase contrast optical micrographs of iPP sheared at the temperature of $145^{\circ} \mathrm{C}$ under the shear rate $56 \mathrm{~s}^{-1}$ with a shear time of $1 \mathrm{~s}$.

structures can be traced back to earlier times (e.g., $120 \mathrm{~s}$ ). These results suggest that, even for the shortest waiting time $\left(t_{w}=60 \mathrm{~s}\right.$ after the first shear time, before they can be seen by optical microscopy), the corresponding small nuclei are present before the second shear "pulse." Furthermore, the number and form of the precursors appears not to change with waiting time for $t_{w}$ of $120 \mathrm{~s}$ or more; therefore, the effect of precursors induced during the first shear pulse on precursor formation during the second shear pulse might be insensitive to $t_{w}$.

Second Step of Shearing. The time evolution of morphology after the second step of shearing for relatively long quiescent time intervals indicates that precursors created during the first pulse of shear are 'labeled' by a thick overgrowth of kebabs, allowing them to be readily distinguished from the additional precursors formed during the second shear pulse (Figures 13 and 14, enlarged images are shown in the rightmost column. To distinguish the nuclei formed in the first step more clearly, we have binarized the image by setting gray scale 0 below certain value and 255 above certain value). As the quiescent interval is reduced from $t_{w}=4$ to 3 to $2 \mathrm{~min}$, the thickness of the overgrowth of lamellae on the precursors created during the first pulse varies in a manner that is consistent with the total amount of time for kebabs to grow outward from them $\left(t_{w}+t, t\right.$ was measured from the end of the second shear pulse); in contrast, the precursors formed during the second shear interval become visible as the lamellae grow from them as a function of $t$. In the situation of $t_{w}=0$ (Figure 14 bottom), it is not possible to determine whether or not the shish that grow during the second half of the shearing time grow from the nuclei induced by the first $1 \mathrm{~s}$ of shear. The benefit of introducing a quiescent time interval is that it allows us to clearly see that the second $1 \mathrm{~s}$ shear pulse induces shish growth in both directions $(\mathbf{v}$ and $-\mathbf{v})$ from the precursors that formed during the initial $1 \mathrm{~s}$ of shear. Thus, the results strongly support the hypothesis that Janeschitz-Kriegl introduced almost 20 years ago, ${ }^{40}$ that point-like (more recently named "oblong”) ${ }^{41}$ precursors form first, followed by shish growth. While the aggregation type mechanism stating that "the polymer chain between nuclei was pulled taut and formed the long chain nuclei” seems less possible. With further increased time interval, the length of the shish grew from these nuclei is also different, on the average, the longer the time interval we wait, the longer the shish length is. So far from our experiments, all morphological evidence pointing to the direction that point nuclei formed first, and then followed by a shish growth. The correctness of this mechanism that the kinetics going through a transition state or growth state represents itself much more clearly and directly in the shish formation morphology induced by shearing at the intermediate shear rate with long shear time as shown in Figure 15, the morphology obtained in this continuously sheared sample for $11 \mathrm{~s}$ is similar to that with 1 min quiescent time interval as shown in Figure 14. We can observe that all shish growth are initiated from point nuclei which appeared darker in the image (Figure 14) and are all similar to those pointed out by arrows of different color according to the time evolution morphology shown in Figure 15. All these phenomena are in favor of the idea of the "transition state mechanism" or growth mechanism hypothesis for the shish-kebab formation. If we consider the different quiescent time interval is the time allowed for the growth of the initial nuclei formed after the first step of shear, then the morphology of 0 min time interval is the limit of the morphology that nuclei growth of zero time, then the formation process should have no difference from those with a quiescent time interval. So our hypothesis is that the nuclei formed at the end of the first step are growing anyway with or without continued shear (with or without quiescent time). The longer the quiescent time and the stronger the effects of the second step shear will better promoting the shish-kebab growth. The total effect of the quiescent interval must be considered as a part of the shish formation mechanism, and we should discuss this point next.

The Role of Critical Shear Time. As mentioned in this paper before, the shish usually cannot be formed "instantaneously" at shear rate larger than the inverse reptation time or the entangled network stretching time, and need to take certain shear time for its formation (about $6 \mathrm{~s}$ for the shear rate of $36 \mathrm{~s}^{-1}$ as shown in Figure 7, or $1 \mathrm{~s}$ for the shear rate of $56 \mathrm{~s}^{-1}$ in Figure 12). In order for such a phenomenon to happen, a critical strain as suggested by $\mathrm{Li}$ is needed in order for the chain segments between the entanglement points to be stretched, ${ }^{31}$ or some critical mechanic work as suggested by Mykhaylyk has been applied onto the system. ${ }^{30}$ Although such critical factors are necessary to form the initial nuclei, it is not sufficient to explain the growth mechanism of the shish. So far we have given the phenomenological framework of the initial nuclei formation and shish growth mechanism. With that we can explain the transition state (or growth) in more physical terms and consistent with all the experimental observations. To make our point more clear, the shear rate dependence of the morphology was observed at $1 \mathrm{~min}$ after the sample was sheared under the shear rate of $56 \mathrm{~s}^{-1}$ with shear time $1 \mathrm{~s}$ as shown in Figure 16. On the basis of the results shown in Figure 7, one would expect that $2 \mathrm{~s}$ of shear at $36 \mathrm{~s}^{-1}$ would not induce shish (since $3 \mathrm{~s}$ is not sufficient to do so). Interestingly, significant shish formation is observed, despite using a slightly lower shear rate of $31 \mathrm{~s}^{-1}$ when a two-step protocol is used with two $1 \mathrm{~s}$ shear pulses separated by a $60 \mathrm{~s}$ waiting time (Figure 16, middle). As expected for a shear rate between $31 \mathrm{~s}^{-1}$ and $56 \mathrm{~s}^{-1}$, the two step protocol using $43 \mathrm{~s}^{-1}$ also induces shish formation during the second shear pulse (Figure 

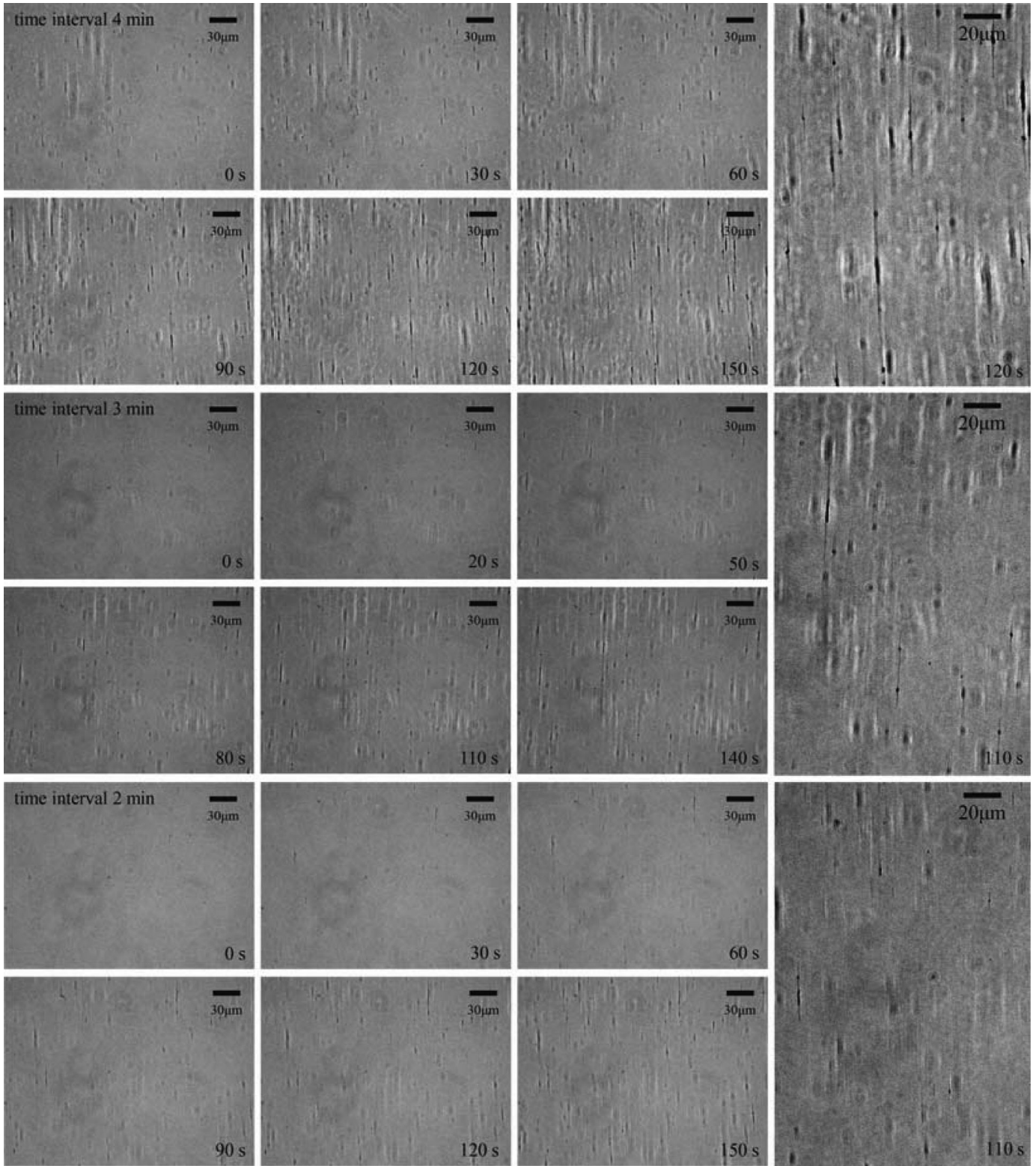

Figure 13. In situ time evolution of PCOM images of iPP after the second step of shearing with quiescent time interval of 4,3 , and 2 min related to the first step. The shear was performed under the same shear condition of the first step (the shear rate of $56 \mathrm{~s}^{-1}$ with shear time $1 \mathrm{~s}$ ). The enlarged images were show at the rightmost column.

16 , bottom). On the other hand, reducing the shear rate to $22 \mathrm{~s}^{-1}$ eliminates the effect (only spherullites indicative of point-like or oblong precursors are evident, Figure 16, top). Those results suggested that there should have existed another relaxation time or characteristic time $\tau_{2}$ associated with the shish formation. In fact, the larger the nuclei become the longer this characteristic time is as have manifested itself in Figures 13 and 14. Therefore, for a given shear time the first stage of the shear which is higher than the critical shear rate, is just to promote the nucleation process. Then these initial nuclei can grow by themselves with or without a continuous shear. After that, either the second stage of the continuous shear or the second step shear offer a quiescent period can promote the large growth of the shish. The characteristic time $\tau_{2}$ (inverse of the second step shear rate needed to promote the shish growth) associated with the second stage of shish growth is obviously related to the size of the gradually growing nuclei (the time of quiescent period or the initial shear time) and the final shear rate applied. Therefore, $\tau_{2}$ should be a function of time and maybe other parameter as well. Note that we may have overly simplified this function by a single characteristic time $\tau_{2}$, but the purpose here is to present a simple physical picture of shish-kebab growth mechanism by identifying this two-step growth process. The role of critical shear time can be also evidenced from the observation of nuclei formed on the interface under a low apparent shear rate $2 \mathrm{~s}^{-1}$, and the waiting for $200 \mathrm{~s}$, after that the shish start to grow from this nuclei as shown in Figure 5. We should note that this $\tau_{2}$ is only a phenomenologically defined characteristic time. The real physical nature can certainly be much more complicated. But for the sake of simple physical interpretation, we choose here to use this phenomenological "time dependent characteristic time $\tau_{2}(\dot{\gamma}, t)$ ", and critical first shear rate $\dot{\gamma} \geq 1 / \tau_{1}$ together with its paired shear time to understand the role of the critical shear time. 


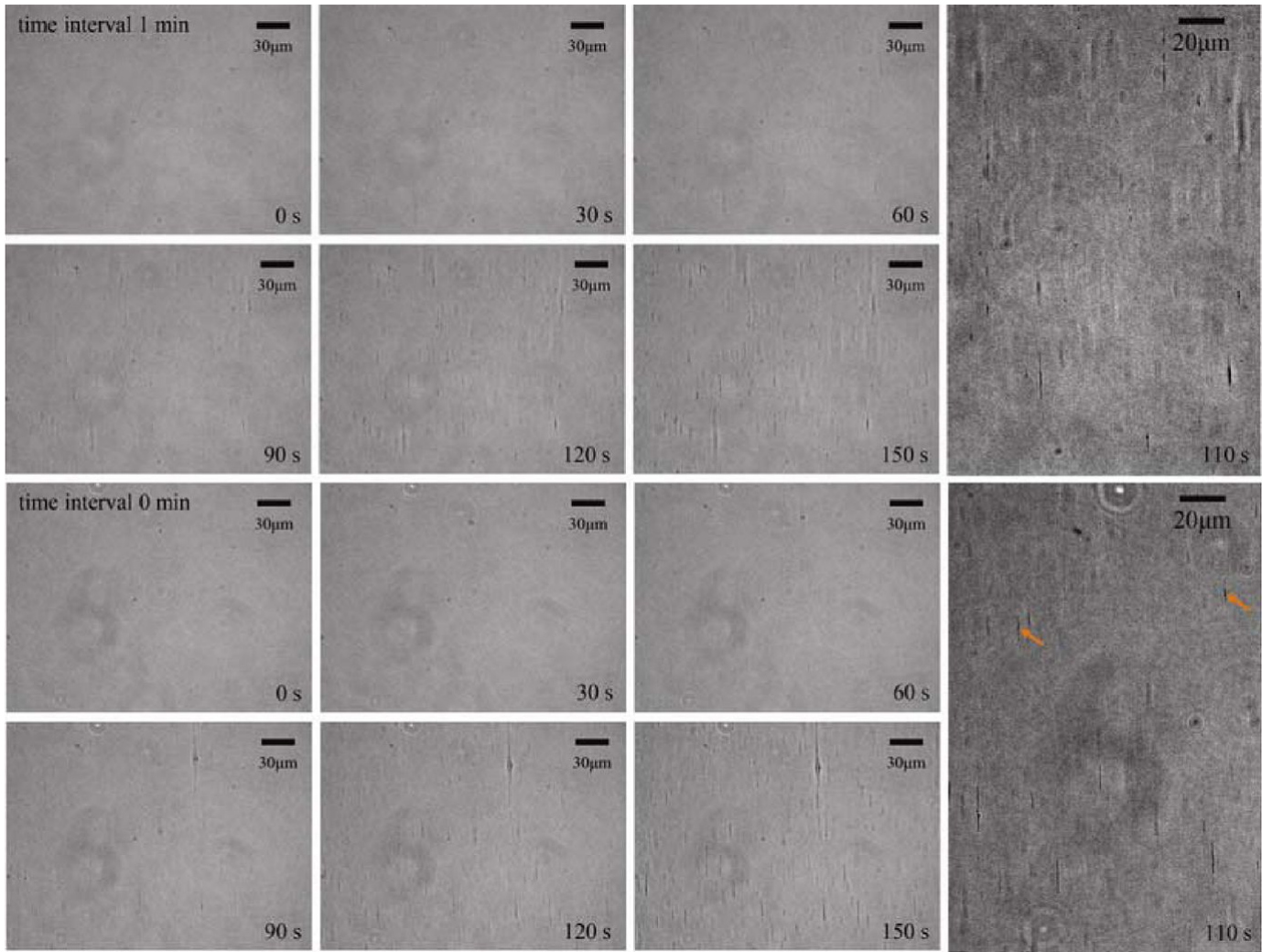

Figure 14. In situ time evolution of PCOM images of iPP after the second step of shearing with quiescent time interval of 1 and 0 min related to the first step. The shear was performed under the same shear condition of the first step (the shear rate of $56 \mathrm{~s}^{-1}$ with shear time $1 \mathrm{~s}$ ). The enlarged images were show at the rightmost column.

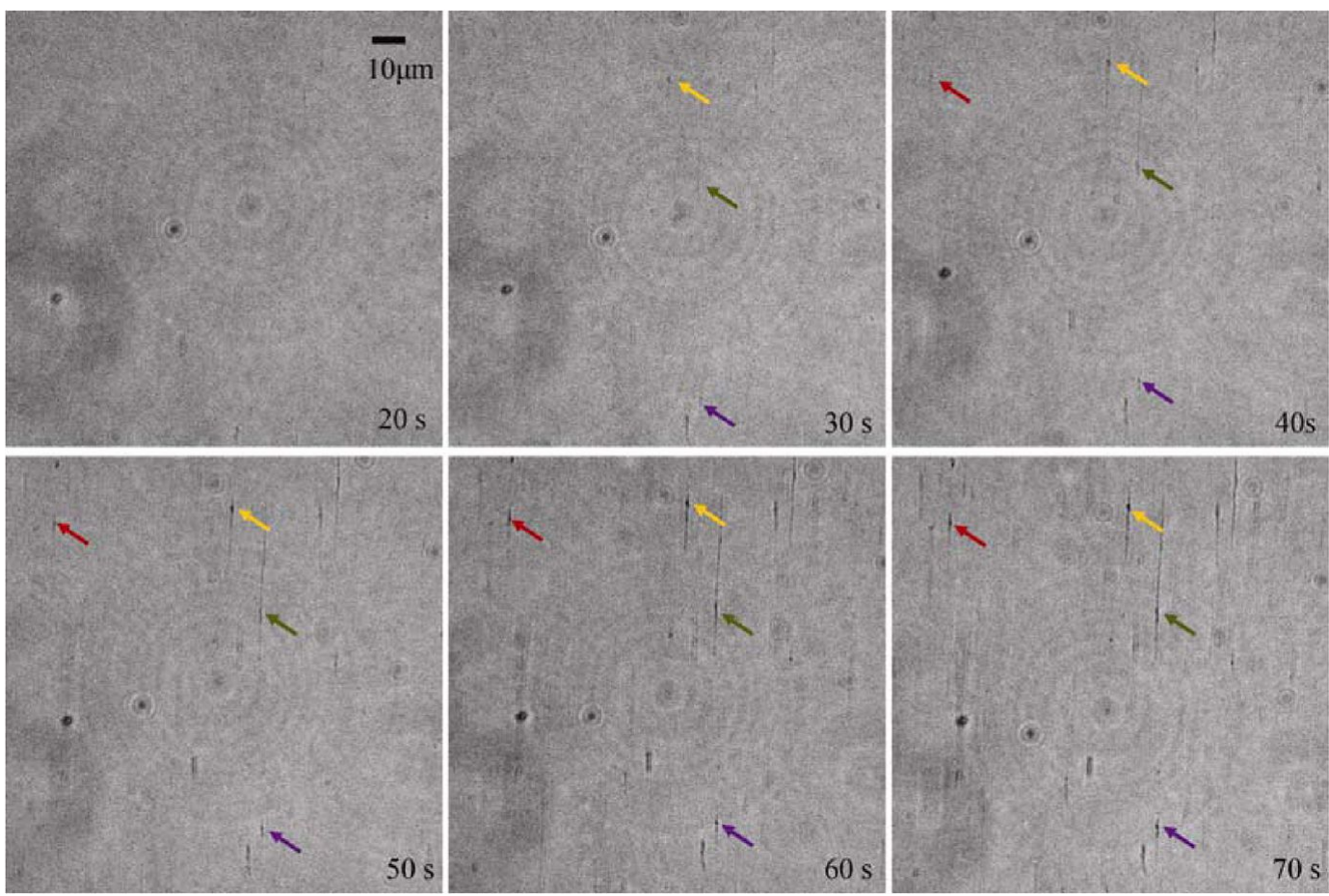

Figure 15. In situ time evolution of phase contrast optical micrographs of iPP sheared at the temperature of $145^{\circ} \mathrm{C}$ under the shear rate $31 \mathrm{~s}^{-1}$ with the shear time of $11 \mathrm{~s}$.

The Mechanism for Shish Formation: Interpret the One Step Shear Morphology Evolution. We like to propose the shish formation mechanism as shown schematically in Figure $17 \mathrm{~b}$ that is interpreted from our in situ morphological study. The final structure of shish-kebab and its growth mechanism must be consistent and connected with each stage. The shear time and shear rate dependence of the shish-kebab growth morphology is the key to understand this important formation process. 

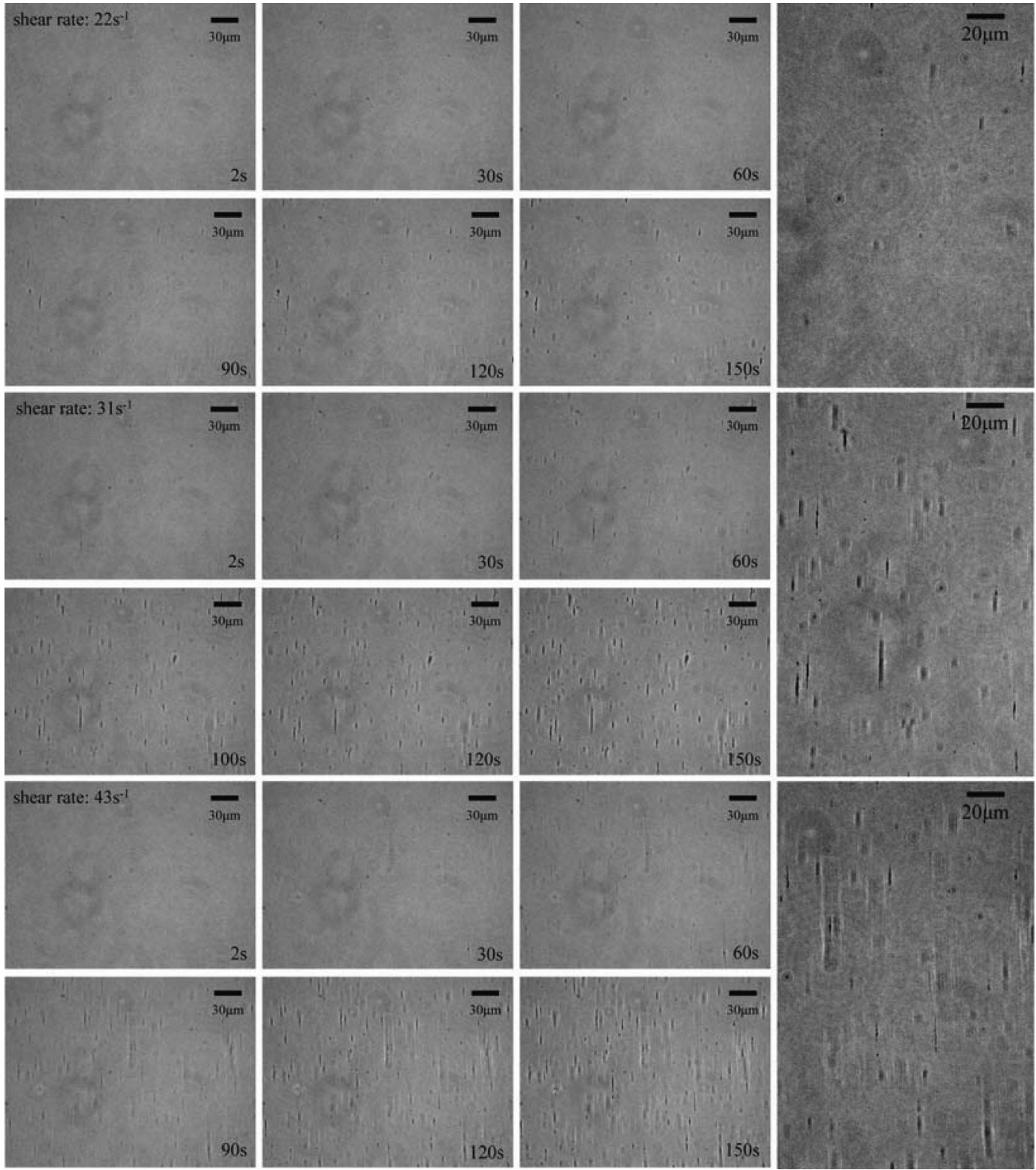

Figure 16. Time evolution morphology of the shear rate dependence $1 \mathrm{~min}$ after the first shear step was done. The enlarged images were show at the rightmost column.

The unstable polymer melt under flow field does not necessarily go directly to the most stable state of the shish-kebab structure. From our observation, it first went through a transient state, and then rapidly transformed into a more stable structure of shishkebab. This transition state separated the whole formation process into two parts: The first part is associated with the point nuclei formation under shear flow with the characteristic time $\tau_{1}$ which might be proportional to the reptation time $\tau_{\mathrm{d}}$ of the polymer or the deformation time of the entangled network. We should also point out that for shear rate smaller than the inverse of $\tau_{1}$, nuclei may also be formed, but those nuclei do not grow into shish-kebab. Although the boundary which define the exact value of the critical shear rate is vague due to the heterogeneous nature of the entangled network or the polydispersity of our sample. But a range of $\tau_{1}$ and the corresponding shear time pair can be defined. The second part is associated with shish formation which should be correlated with the relaxation time or characteristic time $\tau_{2}(\dot{\gamma}, \mathrm{t})$, noticed that $\tau_{2}$ is no longer a constant which should increase with increased quiescent time interval or time from the end of the first stage of shear with the initial value $\tau_{20}$, and depends on the shear rate imposed. The possible way to interpret such time dependent relaxation time $\tau_{2}(\dot{\gamma}, \mathrm{t})$ is correlated with growing nuclei size or the growing shish dimension, with the arms retraction time of all polymer chains already connected to this shish crystal, or with the network deformation time related to the stretching of the network from multiple nuclei under shear deformation. We should notice that although the shear rate is not changed, the $\tau_{2}$ is still a function of time (or quiescent waiting time), and if we gave enough shear time or waiting time for $\tau_{2}$ to become large enough, and then applying the second stage of shear such that the dimensionless flow rate which is the product of the shear rate and the network relaxation time: $\dot{\gamma} \cdot \tau_{2}(\dot{\gamma}, t)>1$, the nucleus formed at the beginning of shear is no longer stable to grow to a spherulite instead it will grow into a shish. Since only the nuclei formed in the earlier time have the chance to satisfy such condition, thus 


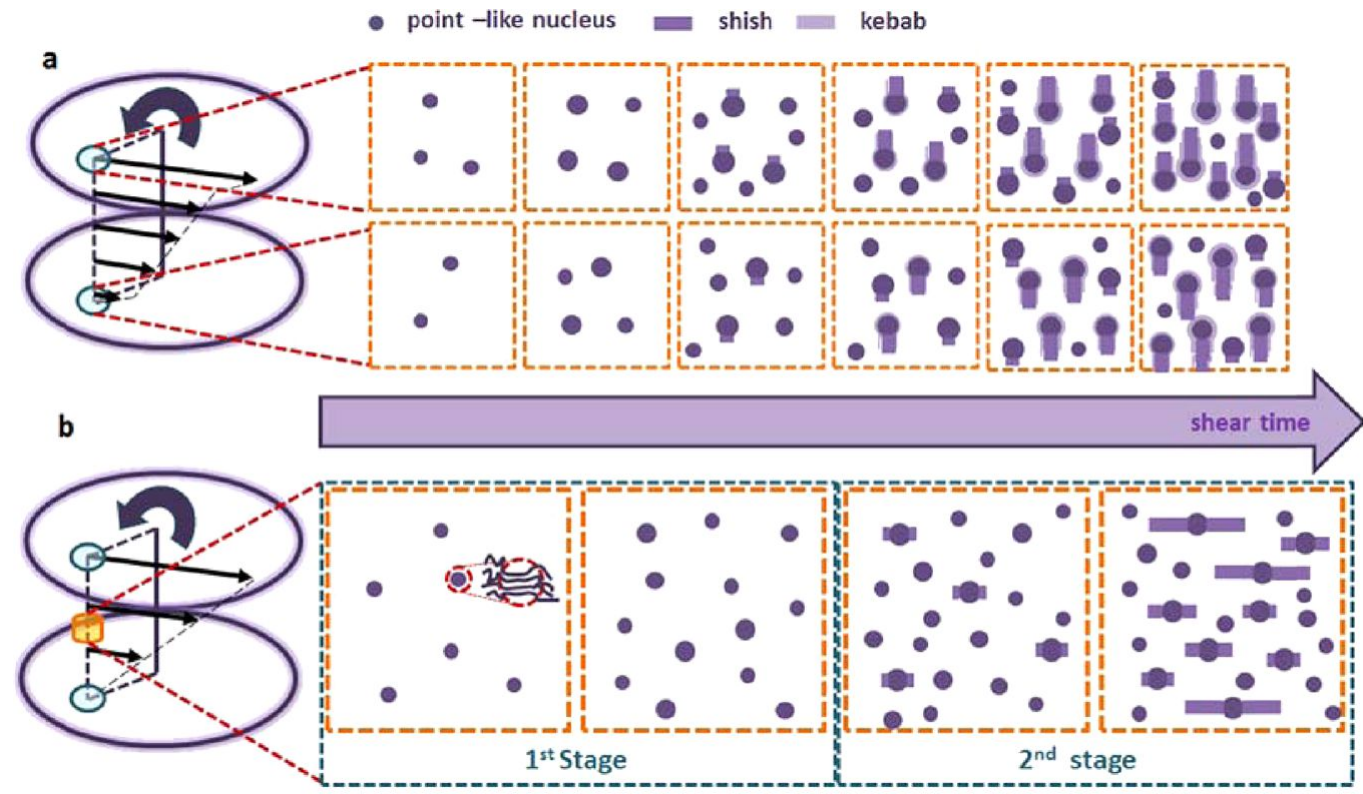

Figure 17. Schematic illustration of transition state mechanism for shish formation: (a) on the interface under the low shear rate while with long shear time; (b) inside of the sample. Refer to the text for more details.

this leads to the gradually changed morphology from spherulitic into cylindrulites as shear time increased. The monotonically increased time dependent $\tau_{2}$ is the reason shish growth can be promoted with smaller and smaller shear rate for the second step shear (as shown in Figure 16) with nuclei growth time. We still do not know the effect of increased $\tau_{2}$ is simply due to the size effect of the nuclei or a cooperative effect such that multiple nuclei under shear could stretch taut the chain segments between entanglement points or between nucleation points and promote the long shish formation.

\section{CONCLUSION}

The comet like morphology of shish-kebab formed at the sample interface and the morphology of shish-kebab with one end initiated from the foreign impurity under shear has demonstrated and captured the main features of the formation of shish-kebab structure: their growth all started from a single point. Moreover these two typical morphologies provided us with a real space visualization of the shish formation mechanism which could be also applied to the interior part of the sample which can go through a transition nucleation stage and then a growth stage for the shish formation. The study on the individual shish-kebab formation by two step shear experiment has provided further key information on such growth kinetics: the initial nuclei formed in the first stage can grow with or without continued shear which will promote the shish growth after the second stage of shear. The direct consequence of such fact is the existence of the critical shear time and our introducing of the second phenomenological time dependent relaxation time $\tau_{2}(\dot{\gamma}, t)$. All the morphological evidence from our experiments are consistent and connected with each other, pointing to one direction: the point nuclei formed first, then followed by the shish growth. Such formation process represents itself more directly and clearly from the morphology of low shear rate experiment with long shear time. The transition state mechanism was proposed without speculation, and consistently captured all the morphological features well, including each typical morphology under various shear rate and shear time in our study.

\section{ASSOCIATED CONTENT}

\section{S Supporting Information}

Discussion of Figures 9and 10 and Figure S1, time evolution of phase contrast microscope observations, showing a similar result. This material is available free of charge via the Internet at http:// pubs.acs.org.

\section{AUTHOR INFORMATION}

\section{Corresponding Author}

*Telephone: +86 10 82618089. Fax: +86 10 62521519. E-mail: c. c.han@iccas.ac.cn.

\section{Notes}

The authors declare no competing financial interest.

\section{ACKNOWLEDGMENTS}

This work has been financially supported by NSFC Project 50930003.

\section{REFERENCES}

(1) Somani, R. H.; Yang, L.; Zhu, L.; Hsiao, B. S. Polymer 2005, 46 (20), 8587-8623.

(2) Kornfield, J. A.; Kumaraswamy, G.; Issaian, A. M. Ind. Eng. Chem. Res. 2002, 41 (25), 6383-6392.

(3) Janeschitz-Kriegl, H.; Ratajski, E. Colloid Polym. Sci. 2010, 288 (16-17), 1525-1537.

(4) Janeschitz-Kriegl, H.; Ratajski, E.; Stadlbauer, M. Rheol. Acta 2003, 42 (4), 355-364.

(5) Li, L. B.; de Jeu, W. H. Flow-induced mesophases in crystallizable polymers. In Advances in Polymer Science; Abe, A., Ed.; Springer-Verlag: Berlin, 2005; Vol. 181, pp 75-120.

(6) Kanaya, T.; Matsuba, G.; Ogino, Y.; Nishida, K.; Shimizu, H. M.; Shinohara, T.; Oku, T.; Suzuki, J.; Otomo, T. Macromolecules 2007, 40 (10), 3650-3654.

(7) Murase, H.; Ohta, Y.; Hashimoto, T. Macromolecules 2011, 44 (18), $7335-7350$.

(8) Balzano, L.; Kukalyekar, N.; Rastogi, S.; Peters, G. W. M.; Chadwick, J. C. Phys. Rev. Lett. 2008, 100 (4), 048302.

(9) Coppola, S.; Grizzuti, N.; Maffettone, P. L. Macromolecules 2001, 34 (14), 5030-5036. 
(10) Zuidema, H.; Peters, G. W. M.; Meijer, H. E. H. Macromol. Theor. Simul. 2001, 10 (5), 447-460.

(11) Zheng, R; Kennedy, P. K. J. Rheol. 2004, 48 (4), 823-842.

(12) Steenbakkers, R. J. A.; Peters, G. W. M. J. Rheol. 2011, 55 (2), 401-433.

(13) Dukovski, I.; Muthukumar, M. J. Chem. Phys. 2003, 118 (14), 6648-6655.

(14) Graham, R. S.; Olmsted, P. D. Phys. Rev. Lett. 2009, 103 (11), 115702.

(15) Jabbarzadeh, A.; Tanner, R. I. Macromolecules 2010, 43 (19), $8136-8142$.

(16) Ogino, Y.; Fukushima, H.; Takahashi, N.; Matsuba, G.; Nishida, K.; Kanaya, T. Macromolecules 2006, 39 (22), 7617-7625.

(17) Somani, R. H.; Yang, L.; Hsiao, B. S.; Sun, T.; Pogodina, N. V.; Lustiger, A. Macromolecules 2005, 38 (4), 1244-1255.

(18) De Gennes, P. G. J. Chem. Phys. 1974, 60 (12), 5030-5042.

(19) Matsuba, G.; Sakamoto, S.; Ogino, Y.; Nishida, K.; Kanaya, T. Macromolecules 2007, 40 (20), 7270-7275.

(20) Jay, F.; Haudin, J. M.; Monasse, B. J. Mater. Sci. 1999, 34 (9), 2089-2102.

(21) Keum, J. K.; Zuo, F.; Hsiao, B. S. Macromolecules 2008, 41 (13), 4766-4776.

(22) Balzano, L.; Rastogi, S.; Peters, G. Macromolecules 2011, 44 (8), 2926-2933.

(23) Seki, M.; Thurman, D. W.; Oberhauser, J. P.; Kornfield, J. A. Macromolecules 2002, 35 (7), 2583-2594.

(24) Fernandez-Ballester, L.; Thurman, D. W.; Zhou, W.; Kornfield, J. A. Macromolecules 2012, 45 (16), 6557-6570.

(25) Kimata, S.; Sakurai, T.; Nozue, Y.; Kasahara, T.; Yamaguchi, N.; Karino, T.; Shibayama, M.; Kornfield, J. A. Science 2007, 316 (5827), 1014-1017.

(26) Janeschitz-Kriegl, H. Crystallization Modalities in Polymer Melt Processing: Fundamental Aspects of Structure Formation; Springer: New York, 2010, pp 1-222

(27) Hoffman, J. D. Polymer 1979, 20 (9), 1071-1077.

(28) Phillips, A. W.; Bhatia, A.; Zhu, P. W.; Edward, G. Macromolecules 2011, 44 (9), 3517-3528.

(29) Heeley, E. L.; Fernyhough, C. M.; Graham, R. S.; Olmsted, P. D.; Inkson, N. J.; Embery, J.; Groves, D. J.; McLeish, T. C. B.; Morgovan, A. C.; Meneau, F.; Bras, W.; Ryan, A. J. Macromolecules 2006, 39 (15), 5058-5071.

(30) Mykhaylyk, O. O.; Chambon, P.; Impradice, C.; Fairclough, J. P. A.; Terrill, N. J.; Ryan, A. J. Macromolecules 2010, 43 (5), 2389-2405.

(31) Yan, T. Z.; Zhao, B. J.; Cong, Y. H.; Fang, Y. Y.; Cheng, S. W.; Li, L. B.; Pan, G. Q.; Wang, Z. J.; Li, X. H.; Bian, F. G. Macromolecules 2010, $43(2), 602-605$.

(32) Zhang, C.; Hu, H.; Wang, D.; Yan, S.; Han, C. C. Polymer 2005, 46 (19), 8157-8161.

(33) Meng, K.; Dong, X.; Zhang, X. H.; Zhang, C. G.; Han, C. C. Macromol. Rapid Commun. 2006, 27 (19), 1677-1683.

(34) Meng, K.; Dong, X.; Hong, S.; Wang, X.; Cheng, H.; Han, C. C. J. Chem. Phys. 2008, 128 (2), 024906.

(35) Mykhaylyk, O. O.; Chambon, P.; Graham, R. S.; Fairclough, J. P.

A.; Olmsted, P. D.; Ryan, A. J. Macromolecules 2008, 41 (6), 1901-1904.

(36) Balzano, L. G.; Rastogi, S.; Peters, G. W. M. Macromolecules 2009, 42 (6), 2088-2092.

(37) Shen, B.; Liang, Y. R.; Zhang, C. G.; Han, C. C. Macromolecules 2011, 44 (17), 6919-6927.

(38) van Meerveld, J.; Peters, G. W. M.; Hutter, M. Rheol. Acta 2004, 44 (2), 119-134.

(39) Ratajski, E.; Janeschitz-Kriegl, H. Polym. Bull. 2012, 68 (6), 17231730.

(40) Liedauer, S.; Eder, G.; Janeschitzkriegl, H. Int. Polym. Proc. 1995, 10 (3), 243-250.

(41) Janeschitz-Kriegl, H.; Ratajski, E. Int. Polym. Proc. 2011, 26 (4), $460-463$. 